DOI: 10.18413/2313-8955-2017-3-3-41-50

УДК [616.37 - 008.64.6: 616.12 - 005.4] - 037: 616.13.002.2 - 004.6 - 073.432.19

Журавлёва Л.В., Лопина Н.А.
ЗНАЧЕНИЕ ТОЛЩИНЫ КОМПЛЕКСА ИНТИМА-МЕДИА СОННЫХ АРТЕРИЙ В ПРОГНОЗИРОВАНИИ АТЕРОСКЛЕРОТИЧЕСКОГО ПОРАЖЕНИЯ КОРОНАРНЫХ СОСУДОВ

Харьковский национальный медицинский университет, проспект Ленина 4, г. Харьков, 61022, Украина. E-mail: prof.zhuravlyova@gmail.com

Аннотация. Цель исследования - оценить толщину комплекса общей сонной артерии (ТИМ ОСА) у больных ишемической болезнью сердца (ИБС) в зависимости от наличия сахарного диабета (СД) 2-го типа и характера поражения коронарных артерий (КА), а также её значение в прогнозировании наличия и выраженности атеросклеротического поражения коронарных сосудов. Maтериальы u методы: обследован 131 пациент с ИБС (89 мужчин, 42 женщин), средний

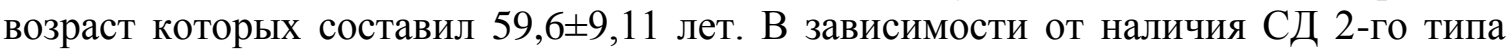
больные ИБС были разделены на 2 группы: 1-ая группа $(\mathrm{n}=70)$ - больные с сопутствующим СД 2-го типа, 2-ая группа $(\mathrm{n}=61)$ - больные ИБС без сопутствующего СД 2-го типа. Всем пациентам для верификации диагноза ИБС проводилась коронарография. У всех больных оценивалась ТИМ ОСА при помощи ультразвуковой допплерографии. Контрольную группу составило 20 практически здоровых добровольцев соответствующего пола и возраста. Результаты. В ходе исследования было выявлено, что у пациентов с ИБС как с сопутствующим СД 2-го типа, так и без были повышены значения ТИМ ОСА достоверно в сравнении с группой контроля $(\mathrm{p}<0.05)$. У лиц с гемодинамически значимыми стенозами КА и диффузным поражением КА как с сопутствующим СД 2-го типа, так и без СД 2-го типа значения ТИМ ОСА были выше, чем у лиц без гемодинамически значимого поражения КА и диффузного поражения КА соответственно. В ходе исследования прогностическая значимость в отношении наличия коронарного атеросклероза установлена для значения ТИМ ОСА более 0,97 мМ, чувствительность и специфичность метода высокие и составляют 89,3\% и 100 \% соответственно,

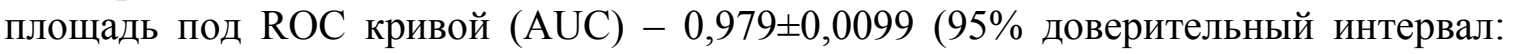
$0,941$ до 0,$995 ; \mathrm{p}<0,0001)$. Прогностическая значимость определения значения ТИМ OCA в отношении наличия гемодинамически значимых стенозов КА установлена для значения ТИМ ОСА более 0,975 мм, чувствительность и специфичность метода составляют 86,7\% и 41,5\% соответственно, площадь под ROC кривой (AUC) $0,584 \pm 0,0546(95 \%$ доверительный интервал: 0,501 до 0,$664 ; \mathrm{p}=0,1239)$. Прогностическая значимость определения значения ТИМ ОСА для прогнозирования наличия диффузного поражения КА установлена для значения ТИМ ОСА более 1,035 мм, чувствительность и специфичность метода составляют $92 \%$ и 39,6\% соответственно, площадь под ROC кривой (AUC) - 0,692 $\pm 0,0428$ (95\% доверительный интервал: 0,612 до 0,765; p<0,0001). Bыводы. Определение ТИМ OCA имеет важное значение как в прогнозировании наличия атеросклеротического поражения коронарных сосудов, так и диагностики гемодинамически значимых стенозов КА, диффузного поражения коронарного русла. 
Ключевые слова: толщина комплекса интима-медиа общих сонных артерий; атеросклероз коронарных сосудов; ишемическая болезнь сердца; сахарный диабет 2-го типа.

L.V. Zhuravlyova, N.A. Lopina

\section{IMPORTANCE OF THE INTIMA MEDIA THICKNESS OF THE COMMON CAROTID ARTERY IN PREDICTION OF ATHEROSCLEROTIC LESIONS OF THE CORONARY VESSELS}

Kharkiv National Medical University, Department of Internal Medicine № 3, 4 Lenin Ave., Kharkiv, 61022, Ukraine.E-mail: prof.zhuravlyova@gmail.com

\begin{abstract}
The purpose of the research was to assess the intima media thickness of the common carotid artery (IMT CCA) in patients with coronary artery disease (CAD), depending on the presence of type 2 diabetes mellitus (T2DM) and the nature of coronary arteries lesions and its value in predicting the presence and the severity of the atherosclerotic lesions of the coronary arteries (CA). Materials and Methods: 131 patients with CAD ( 89 men, 42 women), mean age of $59.6 \pm 9.11$ years were examined. Depending on the presence of T2DM, patients with CAD were divided into 2 groups: $1^{\text {st }}$ group $(n=70)$ - patients with concomitant T2DM, $2^{\text {nd }}$ group $(n=61)-$ patients with CAD without T2DM. Coronarography was performed for all patients to verify the diagnosis of CAD. Also, there were assessed IMT CCA using Doppler ultrasound in all patients. The control group consisted of 20 healthy volunteers of corresponding gender and age.

Results: The study found that patients with CAD both with concomitant T2DM, and with no signs of the disease had increased levels of IMT CCA significantly compared to the control group and the group of comparison $(\mathrm{p}<0.05)$. In persons with hemodynamically significant stenosis of the CA and diffuse damage to the CA with both concomitant DM of type 2 and without type 2 diabetes, the values of IMT CCA were higher than in those without hemodynamically significant CA lesions and diffuse CA lesions, respectively. In our study, the predictive value for the presence of coronary atherosclerosis was set for the value of IMT CCA more than $0.97 \mathrm{~mm}$, the sensitivity and specificity of the method were high and were $89.3 \%$ and $100 \%$ respectively, the area under the ROC curve (AUC) was $0.979 \pm 0.0099(95 \%$ confidence interval: 0.941 to $0.995, \mathrm{p}<0.0001$ ). The prognostic significance of determining the value of IMT CCA for the presence of hemodynamically significant stenosis of the CA was set for the value of the IMT CCA more than $0.975 \mathrm{~mm}$, the sensitivity and specificity of the method are $86.7 \%$ and $41.5 \%$ respectively, the area under the ROC curve (AUC) is $0.584 \pm 0,0546(95 \%$ confidence interval: 0.501 to $0.664, \mathrm{p}=0.1239)$. The prognostic significance of determining the value of IMT CCA for predicting the presence of diffuse coronary artery disease was set for the value of IMT CCA more than $1.035 \mathrm{~mm}$, the sensitivity and specificity of the method are $92 \%$ and $39.6 \%$ respectively, the area under the ROC curve (AUC) is $0.692 \pm 0.0428$ (95\% Confidence interval: 0.612 to $0.765, \mathrm{p}<0.0001$ ). Conclusions. Determination of IMT CCA is important both in predicting the presence of atherosclerotic lesions of the coronary vessels, and in diagnostics of hemodynamically significant coronary artery stenosis, diffuse coronary lesions.
\end{abstract}

Keywords: intima media thickness of the common carotid artery; coronary atherosclerosis; coronary artery disease; type 2 diabetes mellitus.

\section{Введение.} заболевания
Сердечно-сосудистые являются ведущей причиной смертности во всём мире, основой их патогенеза является атеросклеротическое 
поражение сосудов, что приводит к возникновению и прогрессированию ишемической болезни сердца (ИБС) $[1,9]$. В последние годы все больше внимания уделяется активному внедрению профилактических мер для предупреждения CC3, а также разработке новых комплексных диагностических методик, которые можно было бы применять как для скрининга пациентов и своевременной диагностики ИБС, так и для выявления пациентов группы высокого риска [2, 5, 7, 12]. А основу предупреждения СС3 составляет концепция коррекции факторов риска, которая направлена на выявление лиц высокого риска развития СC3 с целью последующего осуществления профилактических мероприятий $[4,5,7,8,14]$. Однако, существующие модели оценки риска - шкалы Framingham Score, PROCAM Score или шкалы Европейского общества кардиологов SCORE, базирующиеся на оценке традиционных факторов риска, не могут в полной мере определить индивидуальный сердечно-сосудистый риск [13]. Особую ценность имеет разработка и уточнение роли существующих маркеров сердечнососудистого риска у лиц низкого риска, исходно считавшихся здоровыми для своевременного начала профилактических мероприятий.

Толщина интима медиа общей сонной артерии (ТИМ ОСА) является неинвазивным маркером изменения артериальной стенки $[10,11]$. Однако недостаточно изучена прогностическая роль ТИМ ОСА при диагностике наличия и выраженности атеросклеротического поражения коронарных артерий (КА).

Цель работы - оценить ТИМ ОСА у больных ишемической болезнью сердца в зависимости от наличия сахарного диабета 2го типа и характера поражения коронарных артерий, a также её значение в прогнозировании наличия и выраженности атеросклеротического поражения коронарных сосудов.

Материалы и методы. В условиях кардиологического отделения КУОЗ
«Областная клиническая больница - Центр экстренной медицинской помощи и медицины катастроф» г. Харькова был обследован 131 пациент с ИБС: стабильной стенокардией напряжения II-III ф.кл. (89 мужчин, 42 женщин), средний возраст которых составил 59,6 $\pm 9,11$ лет. Контрольную группу составило 20 практически здоровых добровольцев соответствующего пола и возраста.

Верификация диагноза - ИБС, стабильная стенокардия напряжения проводился на основании клиникоанамнестического и инструментального исследований путем проведения коронаровентрикулографии, велоэргометрии и холтеровского мониторирования ЭКГ с использованием критериев, рекомендованных Украинским обществом кардиологов (2007), Ассоциации кардиологов Украины (В.M. Коваленко, Н.И.Лутай, Ю.М. Сиренко, 2011), рекомендаций Рабочей группы по проблемам атеросклероза и хронических форм ИБС от 2008 года Ассоциации кардиологов Украины $[5,7]$; диагноз СД - согласно классификации нарушений гликемии (ВО3, 1999) [4, 14]. Верификация диагноза СД 2-го типа основывалась на определении показателей углеводного обмена (использовались показатели краткосрочного и долгосрочного углеводного балансов - гликемический профиль и гликозилированный гемоглобин). Пациенты 1-ой и 2-ой групп были сопоставимы по предшествующему анамнезу приёма статинов (24 пациента 1-ой группы $(34,3 \%)$, и 20 пациентов 2-ой группы $(32,8 \%))$.

Всем пациентам проводилась коронарография правой и левой КА в стандартных проекциях с помощью ангиографа Siemens AXIOM Artis. Также всем пациентам и лицам контрольной группы проводилось допплерографическое исследование сонных артерий с измерением толщины комплекса интима-медиа общих сонных артерий (ТИМ ОСА), определение процента стеноза в зоне максимальной редукции диаметра исследуемой артерии в поперечном сечении, наличия 
атеросклеротической бляшки. Толщина стенки дистальной трети сонных артерий измерялась на расстоянии 1 см проксимальнее бифуркации в В-режиме, в продольном сечении артерии. При этом производили трехкратное измерение величины показателя ТИМ, с каждой стороны определяли среднее, затем вычисляли среднее для правой и левой ОСА. Бляшкой считалась фокальная структура, выступающая в просвет сосуда на 0,5 мм или на 50\% больше величины ТИМ прилегающих участков артерии, или увеличение ТИМ ОСА более 1,3 мм.

В зависимости от наличия СД 2-го типа больные ИБС были разделены на 2 группы: 1-ая группа $(\mathrm{n}=70)$ - больные с сопутствующим СД 2-го типа, 2-ая группа $(\mathrm{n}=61)$ - больные ИБС без сопутствующего СД 2-го типа.

При оценке гемодинамической значимости поражения коронарного русла мы ориентировались на анатомическую классификацию поражений КА, согласно которой стенозы КА менее 70\% принято считать гемодинамически незначимыми, стенозы более $70 \%$ принято считать гемодинамически значимыми $[5,7]$. Следует отметить, что единой классификации гемодинамически значимых стенозов КА не существует, описываются как гемодинамически значимые поражения КА стенозы как более $50 \%$, так и более $70 \%$, и анатомическая классификация, в целом, достаточно условная, так как прежде всего оценка гемодинамической значимости должна базироваться на функциональной оценке. Ввиду того, что оценка функциональной значимости стенозов КА на основании оценки фракционного резерва кровотока (Fractional flow reserve (FFR)), представляющего собой отношение давления дистальнее стеноза к давлению до (проксимальнее) стеноза, в настоящее время недоступна в рутинной клинической практике в большинстве кардиологических клиник нашей страны, анатомическая классификация поражений КА принята в нашем исследовании за единственно возможную и выполнимую в условиях реальной клинической практики для оценки выраженности поражения КА [5, 7].

Bсе пациенты 1-ой и 2-ой групп были разделены на две подгруппы в зависимости от наличия гемодинамически выраженных стенозов КА (стенозирующий атеросклероз КА более 70\%). Пациенты первой группы разделены на - 1а подгруппу ( $\mathrm{n}=19$, стенозы КА менее 70\%), и на 16 подгруппу $(\mathrm{n}=51$, стенозы КА более 70\%), пациенты 2-ой группы разделены на - 2а подгруппу $(\mathrm{n}=15$, стенозы КА менее 70\%), и на 26 подгруппу $(\mathrm{n}=46$, стенозы КА более $70 \%)$. Также для более полной характеристики выраженности поражения КА пациенты делились в зависимости от наличия диффузного поражения КА, что подразумевало собой многососудистое и/или многосегментным поражением КА. В зависимости от наличия диффузного поражения коронарных сосудов пациенты обеих групп были разделены на подгруппы - 1в подгруппа (n=42) - пациенты 1-ой группы с диффузным поражением КА, 1 г подгруппа (n=28) - пациенты 1-ой группы без диффузного поражения КА; 2в подгруппа $(\mathrm{n}=8)$ - пациенты 2-ой группы с диффузным поражением КА, 2 г подгруппа $(\mathrm{n}=53)$ пациенты 2-ой группы без диффузного поражения КА.

Статистическую обработку результатов исследования осуществляли с помощью пакета программ Statistica ver. 10.0 for Windows и Exel 2010. Проверка нормальности распределения проводилась с помощью критерия Вилкоксона, Колмогорова-Смирнова, Шапиро-Уилкса. В случае нормального распределения использовались методы параметрической статистики, при ненормальном распределении оценивались методы непараметрической статистики. Для оценки специфичности и чувствительности диагностической модели применялся ROCанализ с расчётом площади под ROC-кривой. Как интегральный показатель прогностической ценности маркера в диагностике рассчитывалась площадь под ROC-кривой (AUC - Area Under Curv). 
Модель считалась адекватной при площади под кривой более 0,5 при значении $\mathrm{p}<0,05$. Значения AUC 0,5-0,6 оценивались как низкая прогностическая значимость метода диагностики, 0,6-0,7 - средняя прогностическая значимость метода диагностики, 0,7-0,8 - хорошая прогностическая значимость метода диагностики, более $0,8-$ как высокая прогностическая значимость метода диагностики [3, 6].

Оценку достоверности различий между группами при нормальном распределении производили с помощью t-критерия Стьюдента, в случае ненормального распределения с помощью U-критерия Манна - Уитни. Статистически достоверным считали различия при $\mathrm{p}<0,05[3,6]$. Выявлена однородность групп по полу.

Результаты и обсуждение. У пациентов 1-ой группы ТИМ ОСА была достоверно выше в сравнении с группой контроля
$(1,22 \pm 0,10$ мм vs $0,89 \pm 0,06$ мм; $\mathrm{p}=0,00001)$, у пациентов 2-ой группы также ТИМ ОСА была достоверно выше в сравнении с группой контроля $(1,11 \pm 0,15$ мм vs $0,89 \pm 0,06$ мм; $\mathrm{p}=0,00001)$. Кроме того у пациентов 1-ой группы ТИМ ОСА была достоверно выше в сравнении с пациентами 2-ой группы $(1,22 \pm 0,10$ мм vs $1,11 \pm 0,15$ мм; $\mathrm{p}=0,00001)$ (таблица 1).

Также нами проводилась оценка значений ТИМ ОСА у больных ИБС в зависимости от наличия СД 2-го типа у лиц с гемодинамически значимыми и незначимыми стенозами коронарных артерий. $\mathrm{y}$ пациентов 16 подгруппы ТИМ ОСА была достоверно выше, чем у пациентов 1а подгруппы (1,24 $\pm 0,09$ мм vs $1,16 \pm 0,09$ мм; $\mathrm{p}=0,0015) . \quad \mathrm{У}$ пациентов 26 подгруппы ТИМ ОСА была незначительно выше, чем у пациентов 2а подгруппы, при этом разница достоверной не была $(1,11 \pm 0,14$ мм vs $1,08 \pm 0,10$ мм; $p=0,45$ ) (таблица 2).

Таблица 1

Оценка значений ТИМ ОСА у больных ИБС в сравнении с контрольной группой

Table 1

Evaluation of IMT CCA in CAD patients in comparison with the control group

\begin{tabular}{|c|c|c|c|}
\hline Показатель Группы & Контроль $\left(\mathrm{n}_{\mathrm{K}}=20\right)$ & 1 группа $\left(\mathrm{n}_{1}=70\right)$ & 2 группа $\left(\mathrm{n}_{2}=61\right)$ \\
\hline ТИМ ОСА, мм & $0,89 \pm 0,06$ & $1,22 \pm 0,10 * / \#$ & $1,11 \pm 0,15 \# \#$ \\
\hline
\end{tabular}

Примечание: Различие в сравнении с величиной показателя у пациентов 2-ой группы статистически значимо: ${ }^{*} p=0,00001$. Различие в сравнении с величиной показателя у лиц контрольной группы статистически значимо: $\# \mathrm{p}=0,00001 ; \# \# \mathrm{p}=0,00001$.

Таблиия 2

Оценка значений ТИМ ОСА у больных ИБС в зависимости от наличия СД 2-го типа у лиц с гемодинамически значимыми и незначимыми стенозами коронарных артерий

Table 2

Evaluation of IMT CCA in CAD patients, depending on the presence of T2DM in patients with hemodynamically significant and insignificant stenoses of the coronary arteries

\begin{tabular}{|l|c|c|c|c|c|c|}
\hline Показатель Группы & $\begin{array}{c}1 \\
(\mathrm{n}=70)\end{array}$ & $\begin{array}{c}1 \mathrm{a} \\
(\mathrm{n}=19)\end{array}$ & $\begin{array}{c}16 \\
(\mathrm{n}=51)\end{array}$ & $\begin{array}{c}2 \\
(\mathrm{n}=61)\end{array}$ & $\begin{array}{c}2 \mathrm{a} \\
(\mathrm{n}=15)\end{array}$ & $\begin{array}{c}26 \\
(\mathrm{n}=46)\end{array}$ \\
\hline ТИМ ОСА, мм & $1,22 \pm 0,10^{*}$ & $1,16 \pm 0,09$ & $1,24 \pm 0,09 * *$ & $1,11 \pm 0,15$ & $1,08 \pm 0,10$ & $1,11 \pm 0,14$ \\
\hline
\end{tabular}

Примечание: Различие в сравнении с величиной показателя у пациентов 2-ой группы статистически достоверно: * $\mathrm{p}=0,00001$. Различие в сравнении с величиной показателя у пациентов 1а подгруппы группы статистически достоверно: ** $\mathrm{p}=0,0015$.

Также оценивались значения ТИМ ОСА у больных ИБС в зависимости от наличия СД 2-го типа и диффузного поражения коронарных артерий. У пациентов 1в подгруппы ТИМ ОСА была достоверно выше, чем у пациентов 1г подгруппы
$(1,28 \pm 0,05$ мм vs $1,13 \pm 0,07$ мм; *p<0,00001). У пациентов 2в подгруппы ТИМ ОСА была достоверно выше, чем у пациентов 2г подгруппы $(1,26 \pm 0,07$ мм vs $1,08 \pm 0,13$ мм; $\mathrm{p}=0,0003)$ (таблица 3). 


\section{Оценка значений ТИМ ОСА у ИБС в зависимости от наличия СД 2-го типа и диффузного поражения коронарных артерий}

Таблииа 3

Table 3

Evaluation of IMT CCA in CAD, depending on the presence of T2DM and diffuse lesions of the coronary arteries

\begin{tabular}{|c|c|c|c|c|c|c|}
\hline Группы & \multirow{2}{*}{$1(\mathrm{n}=70)$} & \multicolumn{2}{|c|}{$\begin{array}{c}\text { Диффузный характер } \\
\text { поражения }\end{array}$} & \multirow{2}{*}{$2(n=61)$} & \multicolumn{2}{|c|}{$\begin{array}{c}\text { Диффузный характер } \\
\text { поражения }\end{array}$} \\
\hline Показатель & & $\begin{array}{l}\text { Да }-1 в \\
(n=42)\end{array}$ & $\begin{array}{l}\text { Нет }-1 \Gamma \\
(\mathrm{n}=28)\end{array}$ & & $\begin{array}{c}\text { Да }-2 \mathrm{~B} \\
(\mathrm{n}=8)\end{array}$ & $\begin{array}{l}\text { Нет }-2 \Gamma \\
(n=53)\end{array}$ \\
\hline ТИМ ОСА, мм & $1,22 \pm 0,10 *$ & $\begin{array}{l}1,28 \pm \\
0,05^{* *}\end{array}$ & $\begin{array}{c}1,13 \pm \\
0,07\end{array}$ & $1,11 \pm 0,15$ & $\begin{array}{c}1,26 \pm \\
0,07 * * *\end{array}$ & $\begin{array}{c}1,08 \pm \\
0,13\end{array}$ \\
\hline
\end{tabular}

Примечание: Различие в сравнении с величиной показателя у пациентов 2-ой группы статистически достоверно: *p=0,00001. Различие в сравнении с величиной показателя у пациентов 1г подгруппы статистически достоверно: *p<0,00001. Различие в сравнении с величиной показателя у пациентов 2г подгруппы статистически достоверно: ***p=0,0003.

Кроме того, при проведении оценки чувствительности и специфичности значений ТИМ ОСА в прогнозировании атеросклеротического поражения сосудов с помощью продемонстрировано, что информативность в отношении наличия коронарного атеросклероза установлена для значения ТИМ ОСА более 0,97 мм, чувствительность и специфичность метода высокие и составляют 89,3\% и $100 \%$ соответственно, площадь под ROC кривой (AUC) - 0,979 $\pm 0,0099(95 \%$ доверительный интервал: 0,941 до 0,995 ; $\mathrm{p}<0,0001)$ (рис. 1).

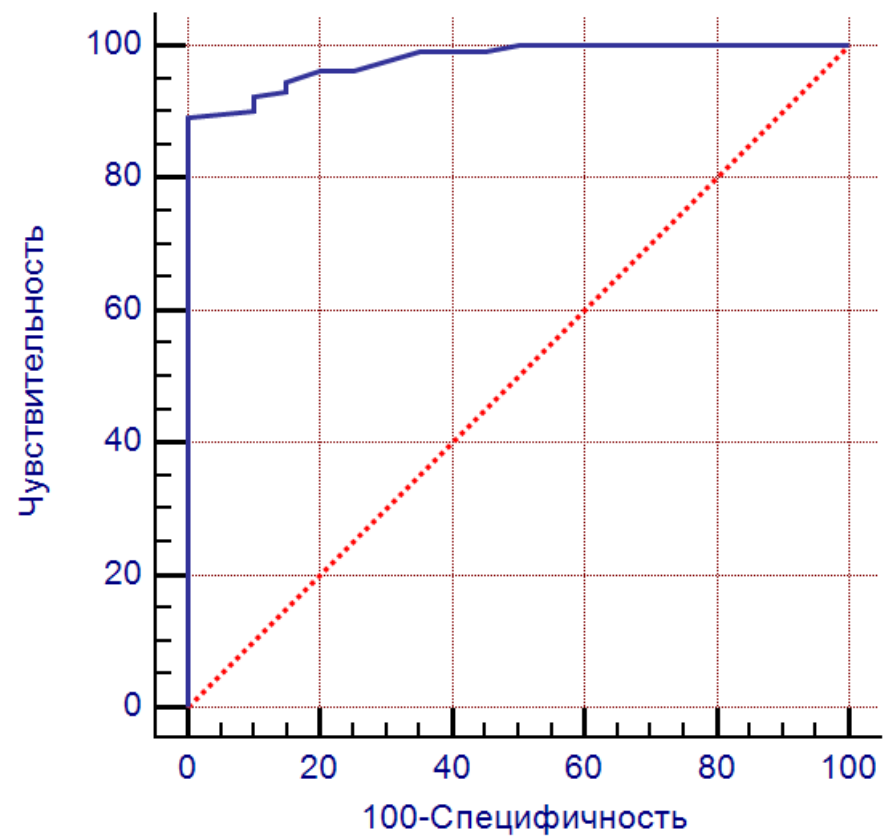

Рис. 1. Чувствительность и специфичность определения значения ТИМ ОСА при выявлении пациентов с атерослеротическим поражением коронарных сосудов. Площадь под кривой $(\mathrm{AUC})=0,979$, станд.ошибка $=0,0099 ; \mathrm{p}<0,0001,95 \%$ ДИ=0,941-0,995

Fig. 1. Sensitivity and specificity of the determination of the value of IMT CCA in the detection of patients with atheroslerotic lesion of coronary vessels. Area under the curve (AUC) $=0.979$, standard error $=0.0099 ; \mathrm{p}<0.0001$, $95 \% \mathrm{CI}=0.941-0.995$ 
Также нами оценивалась диагностическое значение определения ТИМ ОСА в отношении прогнозирования выраженности поражения КА - наличия гемодинамически значимых стенозов и диффузного поражения KA.

В нашем исследовании информативность В отношении наличия гемодинамически значимых стенозов КА установлена для значения ТИМ ОСА более 0,975 мм, чувствительность и специфичность метода составляют $86,7 \%$ и $41,5 \%$ соответственно, площадь под ROC кривой (AUC) 0,584 $\pm 0,0546$ (95\% доверительный интервал: 0,501 до 0,664; $\mathrm{p}=0,1239$ ) (рис.2.).

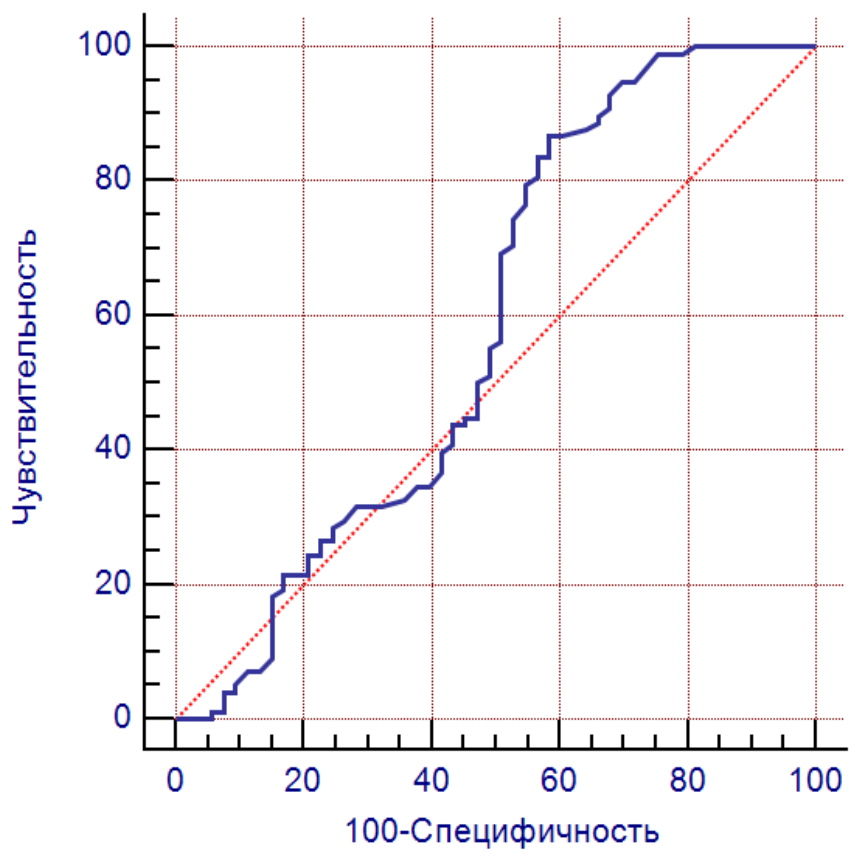

Рис. 2. Чувствительность и специфичность определения значения ТИМ ОСА при прогнозировании наличия гемодинамически значимых стенозов коронарных артерий у пациентов с установленной ИБС. Площадь под кривой $(\mathrm{AUC})=0,584$, станд.ошибка $=0,0546$; $\mathrm{p}=0,1239,95 \%$ ДИ $=0,501-0,664$

Fig. 2. Sensitivity and specificity of the determination of the value of the IMT CCA when predicting the presence of hemodynamically significant stenoses of the coronary arteries in patients with established IHD. Area under the curve $($ AUC $)=0.584$, standard error $=0.0546 ; p=0.1239,95 \% \mathrm{CI}=0.501-0.664$

Прогностическая ценность определения ТИМ OCA у больных с ИБС для прогнозирования наличия диффузного поражения КА выше: информативность в отношении наличия диффузного поражения КА у больных с ИБС установлена для значения ТИМ ОСА более 1,035 мм, чувствительность и специфичность метода составляют $92 \%$ и $39,6 \%$ соответственно, площадь под ROC кривой (AUC) - 0,692 \pm 0,0428 (95\% доверительный интервал: 0,612 до 0,765; $<<0,0001)$ (Рис.3.).

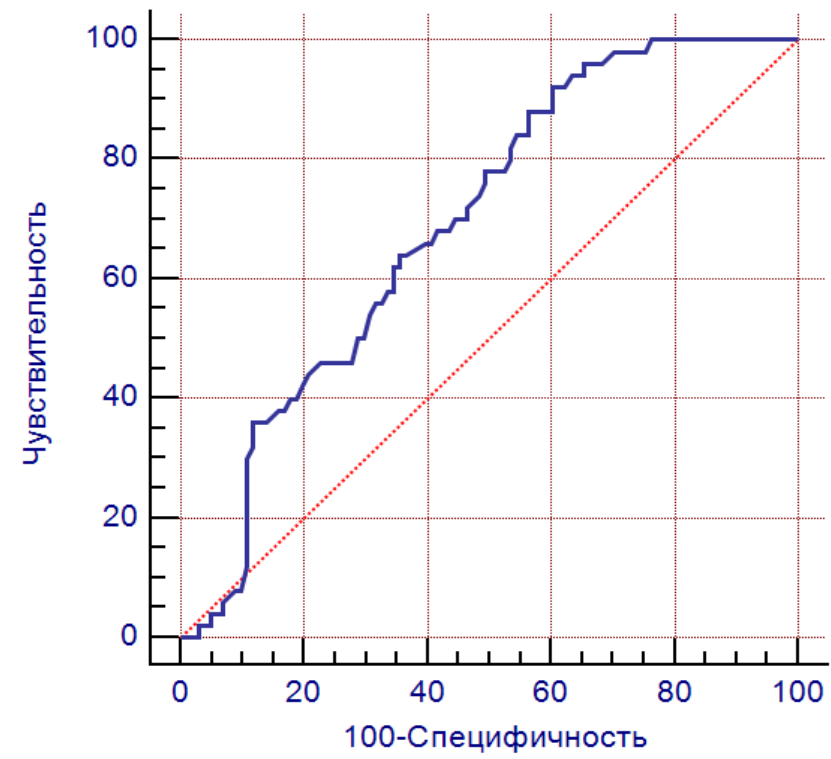

Рис. 3. Чувствительность и специфичность определения значения ТИМ ОСА при прогнозировании наличия диффузного поражения коронарных артерий у пациентов с установленной

ИБС. Площадь под кривой $(\mathrm{AUC})=0,692$, станд.ошибка $=0,0428 ; \mathrm{p}<0,0001$, $95 \%$ ДИ $=0,612-0,765$

Fig. 3. Sensitivity and specificity of the determination of the value of the IMT CCA in predicting the presence of diffuse lesions of the coronary arteries in patients

with established IHD. Area under the curve

$($ AUC $)=0.692$, standard error $=0.0428$;

$$
\mathrm{p}<0.0001,95 \% \mathrm{CI}=0.612-0.765
$$

По данным проведенного нами исследования определение значения ТИМ OCA имеет высокую прогностическую значимость в отношении диагностики наличия коронарного атеросклероза (площадь под $\mathrm{ROC}$ кривой $(\mathrm{AUC})=0,979 \pm 0,0099(95 \%$ доверительный интервал: 0,941-0,995; $\mathrm{p}<0,0001)$ ), установлена для значения ТИМ OCA более 0,97 мм. Прогностическая значимость определения значения ТИМ ОСА в отношении диагностики гемодинамически значимых стенозов низкая (площадь под ROC 
кривой $(\mathrm{AUC})=0,584 \pm 0,0546 \quad(95 \%$ ДИ=0,501-0,664; $\mathrm{p}=0,1239))$. Прогностическая значимость определения значения ТИМ ОСА в отношении диагностики диффузного поражения коронарных артерий средняя (площадь под ROC кривой (AUC) 0,692 $\pm 0,0428$ (95\% доверительный интервал: 0,612-0,765; $\mathrm{p}<0,0001))$, установлена для значения ТИМ ОСА более 1,035 мм.

\section{Выводы}

Таким образом, полученные данные свидетельствуют о повышении ТИМ ОСА, как важного критерия сердечно-сосудистого риска у пациентов с ИБС, особенно на фоне сопутствующего СД 2-го типа и её прогностическую диагностическую ценность, а именно:

1. У пациентов с ИБС как с сопутствующим СД 2-го типа, так и без, повышены значения ТИМ ОСА достоверно в сравнении с группой контроля и сравнения $(\mathrm{p}<0,05) . \quad$ У лиц с гемодинамически значимыми стенозами КА и диффузным поражением КА как с сопутствующим СД 2го типа, так и без СД 2-го типа значения ТИМ ОСА были выше, чем у лиц без гемодинамически значимого поражения КА и диффузного поражения КА соответственно.

2. В ходе исследования прогностическая значимость в отношении наличия коронарного атеросклероза установлена для значения ТИМ ОСА более 0,97 мм, чувствительность и специфичность метода высокие и составляют $89,3 \%$ и $100 \%$ соответственно, площадь под ROC кривой

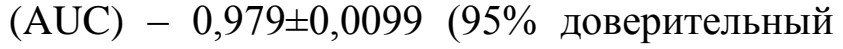
интервал: 0,941 до 0,995; $<<0,0001)$.

3. Прогностическая

значимость определения значения ТИМ ОСА в отношении наличия гемодинамически значимых стенозов КА установлена для значения ТИМ ОСА более 0,975 мм, чувствительность и специфичность метода составляют $86,7 \%$ и 41,5\% соответственно, площадь под ROC кривой (AUC) 0,584 $\pm 0,0546$ (95\% доверительный интервал: 0,501 до 0,664; $\mathrm{p}=0,1239$ ).

4. Прогностическая значимость определения значения

ТИМ ОСА для

прогнозирования наличия диффузного поражения КА установлена для значения ТИМ ОСА более 1,035 мм, чувствительность и специфичность метода составляют 92\% и $39,6 \%$ соответственно, площадь под ROC кривой $\quad(\mathrm{AUC}) \quad-\quad 0,692 \pm 0,0428 \quad(95 \%$ доверительный интервал: 0,612 до 0,765 ; $\mathrm{p}<0,0001)$.

В настоящее время проведение коронарографии, или компьютерной томографии дорогостояще, а проведение скрингового определения ТИМ ОСА, неинвазивного метода визуализации атеросклероза, доступно в рутинной клинической практике, что даёт возможность первичной оценки поражения артериального русла в целом, а как продемонтрировано в проведенном нами исследовании, коронарного бассейна в частности, так как подтверждена высокая прогностическая ценность определения ТИМ ОСА в диагностике наличия и выраженности атеросклеротического поражения КА.

Особенно важное значение имеет оценка ТИМ ОСА для прогнозирования наличия поражения КА у пациентов с сопутствующим СД 2-го типа, когда на фоне диабетической автономной нейропатии имеет место асимптомное или нетипичное течение ИБС, а проведение ХМ-ЭКГ не даёт в полной мере информации о наличия ишемии, а также в случаях, когда проведение нагрузочных тестов противопоказано, а также у лиц с исходно измененной ЭКГ, например полной блокадой левой ножки пучка Гиса с целью отбора пациентов для проведения инвазивной коронарографии. Таким образом, оценка ТИМ ОСА должна стать необходимой составляющей скрининговых программ определения сердечно-сосудистого риска для улучшения стратегий первичной профилактики СС3.

В отношении данной статьи не было зарегистрировано конфликта интересов.

\section{Список литературы}

1. Бідучак А.С., Шкробанець І.Д., Леонець C.I. Епідеміологічні особливості хвороб системи кровообігу в Україні й Чернівецькій області // 
Буковинський медичний вісник. Том 17. № 3 (67). ч. 2. 2013. С. 100-103.

2. Журавлёва Л.В., Лопина Н.А. Уровни фракталкина и асимметричного диметиларгинина у больных ишемической болезнью сердца в зависимости от наличия сахарного диабета 2-го типа и характера поражения коронарных артерий // Научный результат. Медицина и фармация. 2016. №3. C. 11-17.

3. Москаленко В.Ф., Гульчій О.П., Голубчиков М.В., Лєдощук БО., Лєхан В.М., Огнєв В.А. Литвинова Л.О.. Максименко О.П., Тонковид О.Б./ За загальною редакцією членакореспондента АМН України, професора В.Ф. Москаленка / Біостатистика. - К.: Книга плюс, 2009. $184 \mathrm{c}$.

4. Рекомендации по диабету, предиабету и сердечно-сосудистым заболеваниям. EASD/ESC / Российский кардиологический журнал. № 3 (107). 2014. C. 6-70.

5. Стабільна ішемічна хвороба серця: адаптована клінічна настанова, заснована на доказах. - К. 2016. 177 с.

6. Трухачёва Н.В. Математическая статистика в медико-биологических исследованиях применением пакета Statistica. М.: ГЭОТАР Медиа, 2012. 384 с.

7. Уніфікований клінічний протокол первинної та вторинної (спеціалізованої) медичної допомоги: Стабільна ішемічна хвороба серця / Наказ МОЗ України від 02.03.2016. № 152. $61 \mathrm{c}$.

8. Уніфікований клінічний протокол первинної та вторинної (спеціалізованої) медичної допомоги: цукровий діабет 2 типу (наказ МОЗ №1118 від 21.12.2012 р.). 115 с.

9. Huang Y., Cai X., Chen P., Mai W. Associations of prediabetes with all-cause and cardiovascular mortality: A meta-analysis. 2014. Vol.46. Pp.684-692.

10.Mancia G., De Backer G., Dominiczak A. Guidelines for the Management of Arterial Hypertension: The Task Force for the Management of Arterial Hypertension of the European Society of Hypertension (ESH) and of the European Society of Cardiology (ESC). Journal of Hypertension. 2007. Vol.25. Pp.1105 - 1187.

11.Mancia G., Fagard R., Narkiewicz K. ESH/ESC guidelines for the management of arterial hypertension. European Heart Journal. 2013. Vol. 34. Pp. $2159-2219$.

12.Mitchell G.F., Hwang Sh-J., Vasan R.S. Arterial stiffness and cardiovascular events: The
Framingham Heart Study. Circulation. 2010. Vol.121. Vol.4. Pp. 505-11.

13.Sadeghi R., Adnani N., Erfanifar A., Gachkar L., Maghsoomi Z. Premature Coronary Heart Disease and Traditional Risk Factors-Can We Do Better? International Cardivascular Research Journal. 2013. Vol. 7, Suppl.2. Pp.46-50.

14.Standards of medical care in diabetes -2016. American Diabetes Association // Diabetes Care. 2016. Vol. 39, Suppl. 1. Pp.1-109.

\section{References}

1. Biduchak A.C., Shkrobants I.D., Leonets S.I. Epidemiological features of diseases of the circulatory system in Ukraine and Chernivtsi region. Bukovinsky Medical Bulletin. Vol. 17. 3 (67). t. 2. 2013. Pp. 100-103. Ukrainian.

2. Zhuravlyova L.V., Lopina N.A. Fractalkine and asymmetric dimethylarginine levels in patients with coronary artery disease depending on the presence of type 2 diabetes mellitus and the character of coronary arteries lesions. Research Result. Medicine and Pharmacy. 2016. №3. Pp. 11-17. Russian.

3. Moskalenko V.F., Gulchii O.P., Golubchikov M.V., Ledoshchuk B.O., Likhan V.M., Ogniew V.A. Litvinova L.O. Maksimenko O.P., Tonkovid O.B. Ed. By the Corresponding Member of Academy of Medical Sciences of Ukraine, professor V.F. Moskalenk. Biostatistics. K .: Book Plus, 2009. 184 p. Ukrainian.

4. Recommendations for diabetes, prediabetes and cardiovascular diseases. EASD / ESC / Russian Cardiology Magazine. No. 3 (107). 2014. Pp. 6-70. Russian.

5. Stable ischemic heart disease: an adapted clinical approach based on evidence. - K. 2016. 177 p. Ukrainian

6. Tvarkhatova N.V. Mathematical statistics in medical biology studies using the Statistica package. M.: GEOTAR Media, 2012. 384 p. Russian.

7. Unified clinical protocol of primary and secondary (specialized) medical aid: Stable ischemic heart disease. Order of the Ministry of Health of Ukraine dated 02.03.2016. N. 152.61 p. 1. Ukrainian.

8. Unified clinical protocol of primary and secondary (specialized) medical aid: type 2 diabetes mellitus (Ministry of Health Order No. 1118 dated December 21, 2012). 115 p. Ukrainian.

9. Huang Y., Cai X., Chen P., Mai W. Associations of prediabetes with all-cause and 
cardiovascular mortality: A meta-analysis. 2014. Vol.46. Pp.684-692.

10.Mancia G., De Backer G., Dominiczak A. Guidelines for the Management of Arterial Hypertension: The Task Force for the Management of Arterial Hypertension of the European Society of Hypertension (ESH) and of the European Society of Cardiology (ESC). Journal of Hypertension. 2007. Vol.25. Pp.1105-1187.

11.Mancia G., Fagard R., Narkiewicz K. $\mathrm{ESH} / \mathrm{ESC}$ guidelines for the management of arterial hypertension. European Heart Journal. 2013. Vol. 34. Pp. 2159-2219.

12.Mitchell G.F., Hwang Sh-J., Vasan R.S. Arterial stiffness and cardiovascular events: The Framingham Heart Study. Circulation. 2010. Vol.121. Vol.4. Pp. 505-11.

13.Sadeghi R., Adnani N., Erfanifar A., Gachkar L., Maghsoomi Z. Premature Coronary Heart Disease and Traditional Risk Factors-Can We Do Better? International Cardivascular Research Journal. 2013. Vol. 7, Suppl.2. Pp.46-50.

14. Standards of medical care in diabetes 2016. American Diabetes Association // Diabetes Care. 2016. Vol. 39, Suppl. 1. Pp.1-109.

Журавлёва Лариса Владимировна, заведующая кафедрой внутренней медицины № 3, д.м.н., профессор

Лопина Наталия Андреевна, аспирант кафедры внутренней медицины № 3,

Zhuravlyova Larisa Vladimirovna, Head of Department of Internal Medicine № 3, Holder of Habilitation Degree in Medicine, Professor

Lopina Nataliya Andreevna, Postgraduate Student, Department of Internal Medicine № 3 